\title{
Using Unique ORFan Genes as Strain-specific Identifiers for Escherichia Coli
}

\author{
Marta Ferrandis-Vila \\ Friedrich-Loeffler-Institut - Federal Research Institute for Animal Health \\ Sumeet K. Tiwari \\ Robert Koch Institute, Microbial Genomics \\ Svenja Mamerow \\ Friedrich-Loeffler-Institut - Federal Research Institute for Animal Health \\ Torsten Semmler \\ Robert Koch Institute, Microbial Genomics \\ Christian Menge \\ Friedrich-Loeffler-Institut - Federal Research Institute for Animal Health \\ Christian Berens ( $\nabla$ christian.berens@fli.de) \\ Friedrich-Loeffler-Institut - Federal Research Institute for Animal Health
}

\section{Research Article}

Keywords: ORFan gene, strain identification, multiplex PCR, qPCR, E. coli, host-specificity, animal experiment

Posted Date: June 25th, 2021

DOl: https://doi.org/10.21203/rs.3.rs-620539/v1

License: (9) (1) This work is licensed under a Creative Commons Attribution 4.0 International License. Read Full License 


\section{Abstract}

\section{Background}

Bacterial identification at the strain level is a much-needed, but arduous and challenging task. This study aimed to develop a method for identifying and differentiating individual strains among multiple strains of the same bacterial species. The set used for testing the method consisted of 17 Escherichia coli strains picked from a collection of strains isolated in Germany, Spain, the United Kingdom and Vietnam from humans, cattle, swine, wild boars, and chickens. We targeted unique or rare ORFan genes to address the problem of selective and specific strain identification. These ORFan genes, exclusive to each strain, served as templates for developing strain-specific primers.

\section{$\underline{\text { Results }}$}

Most of the strains to be deployed experimentally (14 out of 17) possessed unique ORFan genes that were used to develop strainspecific primers. The remaining three strains were identified by combining a PCR for a rare gene with a selection step for isolating the experimental strains. Multiplex PCR allowed the successful identification of the strains both in vitro in spiked faecal material in addition to in vivo after experimental infections of pigs and recovery of bacteria from faecal material. In addition, primers for qPCR were also developed and quantitative readout from faecal samples after experimental infection was also possible.

\section{Conclusions}

The method described in this manuscript using strain-specific unique genes to identify single strains in a mixture of strains proved itself efficient and reliable in detecting and following individual strains both in vitro and in vivo, representing a fast and inexpensive alternative to more costly methods.

\section{Background}

The tracing of microbes in complex biological systems is indispensable to answer many scientific questions in applied, clinical, and environmental microbiology. Bacterial identification at the strain level is highly challenging since closely related bacteria may have similar morphologic and physiologic profiles, and strains belonging to the same bacterial species are virtually indistinguishable solely by morphological methods (1). Techniques to identify strains have evolved and advanced in the past decades, but all available methods have limitations and flaws, and none of them are $100 \%$ reliable or accurate (3). Differentiation of bacterial strains based on their genome information has become the preferred approach due to its excellent resolution, high reliability, and easy availability (2). Genotyping methods can be grouped into three major categories (2). The first is based on DNA fragment patterns, in which amplification and/or enzymatic digestion of bacterial DNA is followed by electrophoretic resolution of differently sized fragments, the pattern of which serves as specific species or strain identifier. For amplicon-based classification, the DNA sequence of a reference genome has to be at least partially known. Second, DNA-hybridisation systems deploy nucleic acid-based probes, labelled fragments of known sequence complementary to their corresponding targets, which are detected after probe binding. Third, DNA sequence-based genotyping, the most powerful tool currently being used to classify bacteria, utilizes strain-specific variations, such as single nucleotide polymorphisms (SNP) (4) as well as deletion or addition of genetic material (5, 6). These methods all rely on specific differences between individual strains, which have to be at least qualitatively, but preferably quantitatively, detectable within the context of a complex microbial environment, in which one or more strains of the target species may already be present.

Escherichia coli is a commensal member of the vertebrate gut, but certain strains, grouped into $E$. coli pathovars, have acquired virulence genes, mainly located on mobile elements, on multiple occasions resulting in a high degree of genomic flux $(7,8)$. E. coli has an open pan-genome, i.e., the number of genes in the pan-genome (consisting of core and accessory genes) increases with the number of additional genomes sequenced. Many accessory genes are only present in one strain (also known as singletons) or in few strains $(9,10)$. Such genes with no known relatives or homologues in species belonging to other lineages are known as orphan or ORFan genes. (11). Improved genotyping and sequencing techniques during the last two decades have led to the discovery of large numbers of ORFan genes present within bacterial genomes $(11,12)$. 
New studies analysing the genomes of pathogenic and non-pathogenic bacteria have shown that all genomes of a single species have their own share specific and unique ORFan genes, which are common to some strains but are not found consistently in all members of the species: this part of the genome is also considered to belong to the "variable or accessory genome" (18-20). Several ORFan genes are lineage-specific, while others can even be strain-specific and seem to contribute to a particular strain's characteristics, including potential pathogenicity $(21,22)$. These features qualify specific ORFan genes to serve to trace bacteria at the strain level. In this study, we describe an ORFan gene-based identification method characterized by the generation and development of PCR primers specific at the bacterial strain level. An extensive E. coli library containing 1198 whole genomesequenced strains collected by the consortium served as database to identify suitable ORFan genes. Specific regions within these ORFan genes were tested against larger sequence databases to increase the marker's probability of being restricted to a single strain. Specific PCR and quantitative PCR (qPCR) primers derived from the search results were successfully applied in vitro and in vivo in an animal infection experiment.

\section{Materials And Methods}

\section{Strain selection}

One thousand one hundred and ninety-eight E. coli strains were provided by all HECTOR project partners and whole-genomesequenced using short-read technology (van der Putten, B., Tiwari, S.K., Semmler, T. and Schultsz, C., unpublished data). Seventeen strains, carrying extended-spectrum beta-lactamase (ESBL) genes, were selected from this collection for an animal study to assess their colonization properties in livestock animals in parallel with an infection approach using a mixture of strains (cocktail) (Table 1).

To facilitate experimental strain detection in complex, non-sterile matrices by adding a selective culturing step, all 17 strains were artificially selected for rifampicin resistance. To this end, a $50 \mathrm{ml}$ overnight culture of each strain was centrifuged at $4000 \mathrm{xg}$ for $10 \mathrm{~min}$, the pellet resuspended in $1 \mathrm{ml}$ of LB media, and then plated on LB-agar plates containing $100 \mu \mathrm{g} / \mathrm{ml}$ rifampicin (Carl Roth, Karlsruhe, Germany). After overnight incubation at $37^{\circ} \mathrm{C}$, the plates were inspected for colony growth. The rpoB genes of the rifampicin-resistant strains were sequenced and one mutant selected per experimental strain.

\section{ORFan gene identification}

The draft genomes were annotated by Prokka v1.13 (23) using a genus-specific blast for E. coli. The genome was constructed at $95 \%$ amino acid identity by using Roary v3.12.0 (24). Genes found in 99\% of the strains within our collection, were considered to represent core genes and the remaining genes classified as accessory genes. Paralogs were split into different orthologous groups. The strain-specific genes were identified by in-house scripts based on the binary matrix of gene presence or absence obtained from Roary. The nucleotide sequences of these strain-specific genes were extracted for each strain, and their specificity was further confirmed by using BLAST (25). Each strain-specific gene was scanned against the entire gene pool of the HECTOR strain collection. Genes found in other strains or in more than one copy in the same strain at $90 \%$ identity and $90 \%$ coverage were discarded. A gene with a single copy present only in one strain was considered as strain-specific in this study. Its corresponding sequence was extracted based on the respective locus-tag. These genes were further examined individually via online BLAST analysis (26) against the GenBank (25) database specific for $E$. coli in order to assess exclusivity. If the top results from the BLAST (25) analysis of the GenBank (26) database belonged to known plasmids and fully matched the sequences being compared, they were considered plasmid-derived, and excluded from further analysis. If no hits were found from the search, a more exhaustive search against the non-redundant nucleotide database from GenBank was carried out. Those genes with few $(<10)$ or no hits were considered ORFans in this analysis.

\section{PCR and qPCR Primer design}

The ORFan genes detected in silico were used to design primers. If no low-hit ORFans were available for a specific strain, those regions within a selected ORFan gene that showed higher sequence variation when blasted against the GenBank database were used to generate the primers. All PCR and qPCR primers were manually designed for each strain according to the following criteria: primer length and melting temperature, avoidance of dimers, hairpins, and self-complementarity. Specificity verification was performed using the tool Primer-BLAST (26). The PCR primers were designed so that four multiplex PCR reactions could be 
performed in order to qualitatively (i.e., presence/absence from a given sample) trace all 17 experimental strains with a minimum number of reactions. For this, both melting temperatures of the primers and sizes of the products within a multiplex were selected so that the temperature did not differ more than $1^{\circ} \mathrm{C}$ between primer pairs, and product sizes differed by at least $100 \mathrm{bp}$ from each other. The oligonucleotide primers were synthesized by Eurofins Genomics (Ebersberg, Germany). Their sequences are listed in Table 2.

Primers for qPCR were optimized for use with the Luna qPCR mastermix (New England Biolabs Inc., Ipswich, MA, USA). Amplicon sizes ranged between 100 and 250 bp, with a GC content from 40-60\%, a melting temperature not greater than $61{ }^{\circ} \mathrm{C}$ with less than $1{ }^{\circ} \mathrm{C}$ difference between primers of the same pair, and a primer length of 19 to 25 nucleotides. If possible, ORFan gene and ORFan gene region used to design the qPCR primers were the same as the ones used for PCR primer design. The oligonucleotide primers were manufactured by Eurofins Genomics (Ebersberg, Germany). Their sequences are listed in Table 3.

\section{PCR Multiplexes}

Four multiplexes were needed in order to detect all 17 experimental strains. As much as possible, multiplexes targeted strains originating from the same host. In the case of the "Mix multiplex", due either to fragment size or melting temperature three strains were included that didn't fit in the other multiplexes. Multiplex PCR conditions were optimised following the recommendations published by Zangenberg et al. (27) or the PCR mastermix manufacturer. PCR was performed in a total volume of $25 \mu$ containing $2 \mu \mathrm{l}$ of purified DNA, $12.5 \mu \mathrm{l}$ of OneTaq 2x Master Mix with Standard Buffer (New England Biolabs Inc., Ipswich, MA, USA), $9.5 \mu \mathrm{l}$ of nuclease-free water, and $0.5 \mu \mathrm{l}$ each of $10 \mu \mathrm{M}$ forward and reverse primer. The reactions were performed in a Biometra T3 Thermocycler System (Analytik Jena, Jena, Germany) using the conditions specified in Table 4.

Gel electrophoresis was performed by using 1.0\% all-purpose, high-purity agarose (VWR International, Radnor, PA, USA) gels with 0.25X SERVA DNA stain clear G (SERVA Electrophoresis GmbH, Heidelberg, Germany) in 1X Tris-borate-EDTA buffer (VWR International, Radnor, PA, USA) in a Perfect Blue Gel System (VWR International, Radnor, PA, USA) at $150 \mathrm{~V}$ for $1 \mathrm{~h}$. Two microliters of amplified DNA were mixed with $4 \mu \mathrm{l}$ of gel loading dye (New England Biolabs Inc., Ipswich, MA, USA) for analysis. For reference, a Quick-Load Purple 100 bp DNA Ladder (New England Biolabs Inc., Ipswich, MA, USA) was used (bands every 100 bp up to 1000 bp, plus two additional bands at $1200 \mathrm{bp}$ and $1500 \mathrm{bp}$ ).

\section{Quantitative PCR reaction setup}

Quantitative PCR conditions were optimised following the QPCR mastermix manufacturer's specifications. Assays for qPCR were performed on a CFX96 Touch Real-Time PCR Detection System (Bio-Rad Laboratories, Hercules, CA, USA). Reactions contained a total volume of $20 \mu \mathrm{l}$, in which $2 \mu \mathrm{l}$ of purified DNA were used together with $10 \mu \mathrm{l}$ of Luna Universal qPCR Master Mix (New England Biolabs Inc., Ipswich, MA, USA), $7 \mu$ of nuclease-free water, and $0.5 \mu$ each of $10 \mu \mathrm{M}$ forward and reverse primer. Each reaction was performed in triplicate. The cycling conditions included an initial denaturation step of 1 min at $95^{\circ} \mathrm{C}$ followed by 40 cycles of $95^{\circ} \mathrm{C}$ for $15 \mathrm{~s}$ and $60^{\circ} \mathrm{C}$ for $30 \mathrm{~s}$. No-template controls $(2 \mu$ of nuclease-free water instead of DNA extract) and an internal calibrator control for each strain ( $2 \mu \mathrm{l}$ of a DNA concentration with a known Ct value to account for possible variations between plate runs) were performed with each batch of samples tested. The uidA gene encoding a $\beta$-glucuronidase specific for $E$. coli was included in the qPCR assay as housekeeping gene (33).

\section{Specificity and efficiency testing}

Primer pairs were individually tested with their respective target strain first by simplex PCR and afterwards together in multiplexes. For this, each strain was streaked onto a Gassner agar plate (Sifin, Berlin, Germany) containing ceftiofur at $4 \mu \mathrm{g} / \mathrm{ml}$ (ceftiofur hydrochloride, VETRANAL ${ }^{\circledR}$, St. Louis, MO, USA) and allowed to grow overnight at $37^{\circ} \mathrm{C}$. After verifying a pure culture, a single colony of each strain was then picked and used to inoculate $10 \mathrm{ml}$ of fresh LB medium. Liquid cultures were incubated overnight at $37^{\circ} \mathrm{C}$. After $18 \mathrm{~h}, 3 \mathrm{ml}$ of the overnight culture were used to isolate genomic DNA (peqGOLD Bacterial DNA Mini Kit, Peqlab, Erlangen, Germany). A Nanodrop microvolume spectrophotometer (NanoDrop One, Thermo Fisher) was used to monitor DNA quality and concentration. Serial dilutions $(100 \mathrm{ng} / \mu \mathrm{l}$ to $0.1 \mathrm{ng} / \mu \mathrm{l})$ were made from the DNA solutions and used as templates to test primer specificity. Culture mixtures of different strains were also used to ensure no cross-detection occurred. Faecal spiking was performed to verify that the primers were sufficiently specific to detect individual strains in the background of an intestinal 
microbiome. For this, pure cultures of each strain were allowed to grow overnight at $37^{\circ} \mathrm{C}$ in liquid culture. Ten microliters of the overnight culture were used to spike $1 \mathrm{~g}$ of a porcine faecal sample. Single-strain spiking and spiking with mixtures containing 210 of the experimental bacterial strains were performed. Afterwards, DNA was extracted using the Quick-DNA Faecal/Soil Microbe Miniprep Kit (Zymo Research, Irvine, CA, USA).

The efficiency of the qPCR primers was calculated following the recommendations published by Svec et al. (28). Ten-fold dilutions ranging from $10 \mathrm{ng}$ down to $0.0001 \mathrm{ng}$ of DNA were tested in triplicate. The mean average of the triplicates was plotted on a logarithmic scale along with the corresponding template concentrations. A linear regression curve was applied to the data points, and the slope of the trend line was calculated. Finally, efficiency was calculated by using the equation: $E=-1+10^{(-1 / \text { slope })}$. All primer pairs tested showed efficiency values between 90 and $95 \%$. Non-template controls did not show any amplification, and internal calibrator values always stayed within the determined Ct value range.

\section{Tracing of bacterial strains after cocktail infection of piglets}

For in vivo analysis, pigs were inoculated with a bacterial cocktail containing seventeen different strains (Table 1.) The animal experiment was approved by the competent authority (State Office for Agriculture, Food Safety and Fisheries of MecklenburgWestern Pomerania, Rostock, Germany, reference no. 7221.3-1-034/19). Eight German landrace pigs, $42-45$ days old, were purchased from a conventional, high-health pig farm (bhzp Garlitz, Langenheide, Germany) and housed in an environmentally controlled animal facility at the Friedrich-Loeffler-Institut (FLI) on the Isle of Riems, Greifswald. The animals adapted to the environmental conditions for three weeks prior to experimental infection. Meanwhile, faecal samples were collected to determine the resistance status of the coliform bacterial population in the intestinal tract of the pigs. Some samples tested positive for ceftiofur-resistance, but all samples tested negative for ceftiofur/rifampicin double-resistant bacteria. All inoculation strains were grown individually on Gassner plates containing $4 \mu \mathrm{g} / \mathrm{ml}$ ceftiofur and $50 \mu \mathrm{l} / \mathrm{ml} \mathrm{rifampicin}$ and stored at $4{ }^{\circ} \mathrm{C}$. The bacterial cocktail was prepared before inoculation, by mixing liquid cultures of all 17 strains at equal numbers $\left(5.88 \times 10^{8}\right.$ cells per strain) in order to reach a total of $10^{10}$ bacteria per inoculation dose. Mixtures were gently centrifuged, the media removed, and the bacterial pellets resuspended in $10 \mathrm{ml}$ of physiological saline containing $10 \%$ sodium bicarbonate to buffer stomach acid. After re-suspension, cooled individual doses were immediately transported to the animal facility. For inoculation, all animals were lightly sedated intramuscularly with azaperon (Stresnil ${ }^{\circledR}$, Elanco, Greenfield, IN, USA) using $0.5 \mathrm{ml} / 20 \mathrm{~kg}$ of body weight. The inoculation of the strain cocktail was performed intra-gastrically using a gastric tube (B. Braun, Melsungen, Germany). The animals recovered quickly and were fed immediately after the procedure. Post-inoculation, clinical observation of the animals was performed once per day during the entire experiment. Rectal swabs, in addition to faecal samples from the pen, were collected daily from day 1 to 14 post-infection, and every two days from day 15 until day 56 at the end of the experiment.

Rectal swabs were suspended in $1 \mathrm{ml}$ of LB medium and allowed to rest at $37^{\circ} \mathrm{C}$ for $30 \mathrm{~min}$. The swab wash-offs were serially diluted from $10^{-1}$ to $10^{-4}$ and used for plate spotting on Gassner agar plates containing either no antibiotics or ceftiofur $(4 \mu \mathrm{g} / \mathrm{ml})$ and rifampicin $(50 \mu \mathrm{g} / \mathrm{ml})$. For spotting, $10 \mu \mathrm{l}$ droplets of the $10^{-1}$ to $10^{-4}$ dilutions were gently deposited on each plate in duplicate and plates were left open inside the bench for 1-2 minutes to allow excess media to be absorbed by the agar. After overnight aerobic incubation at $37^{\circ} \mathrm{C}$, colonies were counted in each droplet. In addition, $100 \mu$ of the suspension from the rectal swabs were plated on Gassner agar plates containing ceftiofur $(4 \mu \mathrm{g} / \mathrm{ml})$ and rifampicin $(50 \mu \mathrm{g} / \mathrm{ml})$. After overnight incubation at $37^{\circ} \mathrm{C}$, plates were washed off using $2 \mathrm{ml}$ of LB, and the suspension was used to isolate DNA with a commercial kit (peqGOLD Bacterial DNA Mini Kit, Peqlab, Erlangen, Germany).

To collect colonic content and tissue, four animals each were euthanized by intravenous administration of Pentobarbital (Release ${ }^{\circledR} 500 \mathrm{mg} / \mathrm{ml}, \mathrm{WDT}$ ) on days 43 p.i. and 56 p.i., respectively. At post-mortem examination, the intestinal tract of each animal was removed and the colon section was separated by a double ligation. After opening, approximately $50 \mathrm{ml}$ of the content was collected and a large piece of intestinal tissue (approximately 2-3 cm) sampled and gently washed to remove any remaining content.

One gram of content was weighed, diluted in $9 \mathrm{ml}$ of LB medium and allowed to rest at $37^{\circ} \mathrm{C}$ for $30 \mathrm{~min}$. One gram of tissue was weighed, finely chopped, suspended in $9 \mathrm{ml}$ of LB medium and allowed to rest at $37^{\circ} \mathrm{C}$ for $30 \mathrm{~min}$. One hundred microliters of each suspension were serially diluted from $10^{-1}$ to $10^{-4}$ and used for plate spotting (see detailed description above) on Gassner 
agar plates containing either no antibiotics or ceftiofur $(4 \mu \mathrm{g} / \mathrm{ml})$ and rifampicin $(50 \mu \mathrm{g} / \mathrm{ml})$. After overnight incubation at $37^{\circ} \mathrm{C}$, colonies were counted in each droplet.

One millilitre of the initial dilution $\left(10^{-1}\right)$ was used to isolate DNA with a commercial kit (peqGOLD Bacterial DNA Mini Kit, Peqlab, Erlangen, Germany) prior to enrichment.

Sample enrichment was performed by adding rifampicin to the initial content suspensions, which was further incubated overnight at $37^{\circ} \mathrm{C}$. The next day, $100 \mu \mathrm{l}$ of the overnight suspension were plated on Gassner agar plates containing ceftiofur $(4 \mu \mathrm{g} / \mathrm{ml}) \mathrm{and}$ rifampicin $(50 \mu \mathrm{g} / \mathrm{ml})$. After overnight aerobic incubation at $37^{\circ} \mathrm{C}$, plates were washed off using $2 \mathrm{ml}$ of LB. One millilitre of the suspension was used for generating glycerol stocks and the remaining millilitre was used to isolate DNA with a commercial kit (peqGOLD Bacterial DNA Mini Kit, Peqlab, Erlangen, Germany).

\section{Statistical analysis}

Statistical analyses were performed with GraphPad Prism Software (GraphPad Prism version 9.0.2 for Windows, GraphPad Software, San Diego, California USA, www.graphpad.com). The one-way ANOVA on ranks (Kruskal-Wallis) test for multiple comparisons was used to determine the significance of differences between $\Delta \mathrm{Ct}$ ( $\Delta \mathrm{Ct}=\mathrm{Ct}$ (gene of interest) $-\mathrm{Ct}$ (housekeeping gene)) values from strains. Values with $p \leq 0.05$ were considered significant.

\section{Results}

\section{ORFan gene identification}

A total of 299 ORFan genes were identified in the whole genome sequences of the 17 experimental strains (Table 5).

The number of strain-specific genes per strain was highly variable, ranging from a maximum of 158 in one strain to zero in two strains. Approximately one-third (86) of the 299 strain-specific genes were classified as plasmid-borne and, therefore, not deemed suitable for strain identification. They were not further analysed as to their ORFan status. Nearly all (206 out of 213) of the strainspecific genes, that were not potentially plasmid-encoded, turned out to be ORFan genes. For the three remaining strains that showed no ORFan genes, a comparison within the 17 strains selected for the bacterial cocktail was made. Genes that were unique among the cocktail strains were then used as strain identifiers.

\section{PCR primer specificity and multiplex functionality}

A primer pair was designed for each experimental strain (Table 2). Only primer pairs that exclusively amplified DNA of the corresponding strain were considered target-specific. Among the initially designed primers, seven pairs failed (41.2\%), either by not-amplifying their respective target or by yielding non-specific bands when tested against other strains. Consequently, new primers were designed and two pairs again failed (28.5\%) for the same reasons. The third pair of primers yielded specific signals for the last two strains. Combining the primer pairs into multiplexes affected neither their specificity nor their ability to identify the matching strain (Figure 1). No non-specific bands were seen after multiplexing the primers; however, it was observed that the bands of the smallest fragments in the multiplex (smaller than $150 \mathrm{bp}$ ) had reduced intensity in those multiplexes containing a larger number of primer pairs (e.g., Cattle multiplex with six pairs of primers and Pig multiplex with five pairs of primers - see Table 4).

During multiplex testing with DNA isolated from pure cultures, the detection limit of the reactions ranged between 1 to $10 \mathrm{ng} / \mu \mathrm{l}$ DNA, which is equivalent to 200 to 2000 genome copies. However, DNA isolated directly from faecal samples spiked with $10^{8}$ bacteria did not yield positive results, indicating that inhibitory substances or the high endogenous background of bacterial and eukaryotic DNA might be problematic in the aforementioned set up. To overcome this obstacle, a selection step was added for experimental strain detection from faecal samples. Since all experimental strains possessed ESBL genes and were rifampicin resistant, a plating step on Gassner agar containing ceftiofur $(4 \mu \mathrm{g} / \mathrm{ml})$ and rifampicin $(50 \mu \mathrm{g} / \mathrm{ml})$ was added to limit strain isolation to the experimental strains and to eliminate the presence of inhibitory faecal substances. This eliminated growth of 
other endogenous ESBL bacteria that were already present in the animals pre-inoculation, and facilitated specific detection of experimental strains in a multiplex PCR approach using boiled lysates or DNA prepared from the pooled bacteria.

\section{Monitoring of faecal samples}

The suitability of the ORFan approach to qualitatively monitor shedding of different $E$. coli strains by pigs when inoculated with 17 strains simultaneously was assessed by conducting colony counting of resistant $E$. coli and detection of strains by classical PCR. All experimental strains were confirmed to be present in the initial inoculation cocktail prepared to be given to the animals (Figure 2). After inoculation of the animals with the bacterial cocktail, faecal samples were collected for 56 days. Results after the first $24 \mathrm{~h}$ post-inoculation were highly variable between animals. After 48 h, 12 of the 17 strains were detected in at least one animal, with a minimum of four strains and a maximum of twelve strains detected in the eight animals. The remaining five strains were not detected at all in faecal matter during the experiment.

Experimental strains counts, i.e., the total number of colonies, which grew without differentiating the single strains, remained high up to day 3 - 4 post-inoculation, after which they slowly declined. A significant number of strains from the inoculum mixture were detectable at day 3 (Figure 3), with three animals being positive for four strains, one animal for six strains, one animal for nine strains, two animals for eleven strains, and one animal positive for twelve strains. Inoculated bacteria were shed and identified by PCR up to day 21 p.i., when their counts on selective plates had declined to single-digit numbers or were absent, requiring an enrichment protocol for further detection of experimental strains. By day 29 p.i., only 5-6 different strains were shed, even after enrichment, and by day 53 p.i., only four experimental strains were detected.

\section{qPCR system results}

The qPCR system was used to prove the general suitability of the ORFan approach to compare different $E$. coli strains quantitatively within complex intestinal porcine microbiomes. To this end, strains that gave positive signals in the PCR Multiplex setup were further analysed via qPCR for their presence in the dissection sample contents. Delta-Ct $(\Delta \mathrm{Ct})$ values obtained from the qPCRs showed significant differences in quantities between the strains. The strain classified as P3 (39533) showed the lowest $\Delta \mathrm{Ct}$ values, indicating a high presence in the colonic content. This strain's presence in the samples was significantly different from all other strains tested via qPCR (Figure 4; Table 6). The strains classified as C2 (R45) and M1 (21225_2\#178) showed the highest $\Delta \mathrm{Ct}$ values on average, denoting both strains' lower presence in the intestinal content of the inoculated animals. Also, M1, together with P2 (IMT28138), showed the lowest degree of significance when compared against the other strains tested (Figure 4; Table 6).

\section{Discussion}

Current strain identification methods based on DNA fragment patterns, including pulsed-field gel electrophoresis (PFGE), restriction fragment length polymorphisms (RFLP), repetitive sequencing-based PCR (REP-PCR), Enterobacterial Repetitive Intergenic Consensus PCR (ERIC-PCR), and multiple-locus variable-number tandem repeat analysis (MLVA) (2), cannot be used to characterize mixtures of strains because it is not possible to assign individual bands to their cognate isolate unambiguously. DNA sequencing-based methods cannot resolve individual strains in a mixture except if sequences with differentiating SNPs have been identified for all strains. The analysis would then require prior testing of the animals to ensure the absence of these discriminatory SNPs in the endogenous bacterial population. DNA hybridization-based methods, such as CDNA and oligonucleotide microarrays, permit the detection of individual genes or gene fragments and, consequently, strains, but they also require previous knowledge of strain-specific sequences and have to be individually adapted for each new combination of to-be-detected strains. Other strategies, commonly used in infection models, like introducing artificial selection markers, such as antimicrobial resistance genes or genes coding for fluorescent proteins, are of limited feasibility since they allow only a limited number of strains to be introduced simultaneously (31).

The ORFan gene targeting approach utilized herein has the advantage of allowing the introduction of multiple strains simultaneously into an experimental setup, including an in vivo animal trial, as demonstrated. As opposed to existing methods for strain identification, which are either extremely time-consuming, expensive or need specific equipment, the ORFan identification system can be implemented relatively fast and is accessible to everyone with standard laboratory equipment and moderate

Page $7 / 22$ 
bioinformatics knowledge. This method can also be flexibly and easily scaled up, because it only requires the identification of a specific ORFan gene for any novel strain to be introduced.

The ORFan gene approach allows the combination with other visualization techniques to expand strain-specific detection to other types of biological sample. Recently, a FISH (fluorescence in situ hybridization)-based strain identification technique was described (32). It has proved to be very useful at the species identification level, based on 16S rDNA sequence specificity. Using ORFan genes instead of 16S rDNA as targets for such strain-specific fluorescent hybridization probes could generate an alternative and complementary data set on individual strains. Fluorescent detection of ORFan gene presence via hybridization of fixed and permeabilized cells would allow rapid quantification of large samples via fluorescence-activated cell sorting. Another possible application could be specific strain detection in tissue sections from infected animals, which would even permit comparative spatial-temporal resolution of multiple strains in a host coupled with respective niche identification.

The ORFan gene identification workflow pinpointed a large enough number of ORFan genes for most strains to allow the successful selection of specific primers. Previous $E$. coli phylogenetic studies unveiled that ORFan genes could compose approximately $1 \%$ of the bacteria's total core genome, with an increase of approximately 26 genes per new genome sequenced (29). The average number of 18 specific genes per strain was lower for the set of strains selected for the animal test, and the respective total number of strain-specific genes was lower for 15 of the 17 strains. An additional complication is that these genes could also be located on plasmids and, thus, subjected to horizontal transmission, disqualifying them as stable markers. In our study, approximately one-third of the strain-specific genes were classified as plasmid-derived, but all affected strains, except one, harboured alternate ORFans for which a specific detection system could be designed. Primer implementation was an iterative process, requiring up to three rounds of sequence selection until all 17 strains could be explicitly identified in multiplex PCR assays. Each round required up to two weeks of time spent between ORFan gene selection and successful testing of the specific primer pairs. For the three strains, for which no ORFan genes were identified, an additional search of their accessory genomes was performed to identify unique genes within the sequence context of the 17 experimental strains. ORFan genes found using this approach were also subjected to the workflow presented in the "Materials and Methods" section to assess exclusivity. As expected, most of the newly identified genes did have more than 10 hits in the GenBank search. For this reason, only nucleotide stretches within a selected ORFan gene that showed a higher degree of sequence variation between the experimental strain's sequence and the sequences available in GenBank, were used to generate primers. Furthermore, designated cocktail strains were artificially rendered rifampicin-resistant to distinguish them from cephalosporin-resistant $E$. coli in the endogenous animal microbiota. The combination of these measures was successful for expanding the limits of the ORFan approach, at least for the list of bacteria under study and in the group of animals used.

An aliquot from the strain cocktail used to infect the animals was immediately stored at $-80^{\circ}$ after preparation. DNA was extracted, and the four multiplexes performed to corroborate the experimental strains' presence. All 17 strains were detected, confirming their presence in the inoculation cocktail. Their respective band intensities were also similar, indicating that each strain had been added in a similar quantity to the cocktail. Among the 17 strains used to infect the pigs, five strains were not detected in the faecal samples throughout the experiment and were also not detected in the intestinal content at necropsy. Eight strains were only detectable intermittently during the entire experiment, with all of them displaying higher prevalence during the first 14 days p.i.. The remaining four strains were consistently detected throughout the entire experiment. The presence or absence of the 12 successfully detected strains was closely monitored during the entire experiment via the multiplex PCRs, demonstrating that the detection system can be used to follow dynamic changes in a strain's presence.

All qPCR primers tested showed high efficiency with values ranging from 90-95\%. Several tests with various samples corroborated that the primers were indeed sufficiently specific to allow the direct use of faecal DNA to detect experimental bacteria from the mixed background of total bacteria. Strain presence in gut content from the animals' colon, which had already been demonstrated in the multiplex PCRs, was also seen with qPCR primers. The qPCR data indicated colonization differences between experimental strains. Based on the qPCR results, strains with high colonization capacity were also easily re-isolated from the faecal samples, demonstrating that the qPCR results are accurate at monitoring specific strain abundancies in the colonic samples.

Next-generation metagenome sequencing provides vital information on microbial populations and genetic diversity at all taxonomic levels. A fast and easy but robust and reliable method for individual strain identification based on information derived 
from whole-genome sequencing has yet to be described. The usage of ORFan genes, specific for individual strains, could be such a valuable tool, as it allows to develop precise PCR markers for tracing the strains in complex mixed-culture experiments. The method has the potential to be applied in multiple ways to foster our understanding of, for example, the population dynamics of closely related strains of a pathogen. A probiotic or any other strain of interest can be evaluated as to its colonization ability, general strain fitness, or zoonotic risk and might guide the development of intervention strategies (2). This method could also be potentially used as a powerful tool for the rapid identification of specific pathogenic strains in the event of an outbreak. Without the need to amplify a whole set of virulence markers characteristic of an outbreak strain, ORFans may be used for quick and specific-strain identification via PCR, such as in different sample types with mixed backgrounds. This would allow to perform large-scale surveys of many different samples to identify potential outbreak sources and clusters. Similar approaches have been used in the past, such as the one described by Bielaszewska et al. (2011), where an outbreak strain was successfully identified by detecting a specific virulence gene profile (34). A similar approach was described by Kiel et al. (2018), where two pipelines were simultaneously run, comparing Shiga-toxin expressing E. coli (STEC) genomes versus control genomes and an STEC core proteome versus control proteomes. Lineage- and serotype-specific genes were identified this way and used for monitoring specific STEC strains (35).

\section{Conclusion}

The method described in this manuscript using single unique genes to identify specific strains proved easy to implement and very reliable in identifying and following individual strains and their dynamics in an in vivo animal model of experimental infection, thus representing a fast, inexpensive and reliable alternative to more costly and laborious identification methods.

\section{Abbreviations}

$\Delta \mathrm{Ct}$ - Delta threshold cycle

BLAST - basic local alignment search tool

bp - Base pair

Ct - Threshold cycle

EDTA - Ethylenediaminetetraacetic acid

ERIC-PCR - Enterobacterial Repetitive Intergenic Consensus PCR

ESBL - Extended-spectrum beta-lactamase

FISH - Fluorescence in situ hybridization

FLI - Friedrich Loeffler Institute

LB - Lysogeny broth

MLVA - Multiple-locus variable-number tandem repeat analysis

ORFan - Open reading frames

PCR - Polymerase chain reaction

p.i. - Post-inoculation

PFGE - Pulsed-field gel electrophoresis

qPCR - Quantitative polymerase chain reaction

Page 9/22 
REP-PCR - Repetitive sequencing-based PCR

RFLP - Restriction fragment length polymorphisms

SNP - Single-nucleotide polymorphism

STEC - Shiga-toxin expressing E. coli

\section{Declarations}

\section{Ethics approval and consent to participate}

The experimental animal study involving pigs was approved by the competent authority (State Office for Agriculture, Food Safety and Fisheries of Mecklenburg-Western Pomerania, Rostock, Germany) under the reference no. 7221.3-1-034/19-1.

All experiments involving animals were performed according to the guidelines for the care and use of experimental animals established by the State Office for Agriculture, Food Safety and Fisheries of Mecklenburg-Western Pomerania.

The study was carried out in compliance with the ARRIVE guidelines.

\section{Consent for publication}

Not applicable.

\section{Availability of data and materials}

The datasets generated and/or analysed during the current study are available in the NCBI repository, under the title "The impact of Host restriction of Escherichia coli on Transmission dynamics and spread of antimicrobial Resistance", BioProject number PRJNA739205.

\section{Competing interests}

The authors declare that they have no competing interests.

\section{Funding}

The HECTOR research project was supported under the framework of the JPIAMR - Joint Programming Initiative on Antimicrobial Resistance - through the 3rd joint call, thanks to the generous funding by the Netherlands Organization for Health Research and Development (ZonMw, grant number 547001012), the Federal Ministry of Education and Research (BMBF/DLR grant numbers 01KI1703A, 01KI1703B and 01 KI703C), the State Research Agency (AEI) of the Ministry of Science, Innovation and Universities (MINECO, grant number PCIN-2016-096), and the Medical Research Council (MRC, grant number MR/R002762/1). The funders were not involved in (i) the design of the study, (ii) the collection, analysis, and interpretation of data and (iii) in writing the manuscript.

\section{Authors' contributions}

$\mathrm{CB}, \mathrm{MFV}$ and CM designed the study, TS and SKT analysed data, MFV and SM performed the experiments, CB and MFV interpreted the data, CB and MFV drafted the manuscript, which was substantially revised by SM, CM, TS, SS and SKT. All authors approved the final version.

\section{Acknowledgements}

HECTOR consortium members:

Alvarez, Julio (VISAVET, Health Surveillance Center and Animal Health Department, Veterinary Faculty, Complutense University of Madrid, Spain). 
Bethe, Astrid (Institute for Microbiology and Epizootics, Freie Universität Berlin, Berlin, Germany).

Bootsma, Martin (Mathematical Institute and UMCU, Utrecht University, Utrecht, the Netherlands).

Fivian-Hughes Amanda (University of Surrey, School of Veterinary Medicine, Guildford, Surrey, United Kingdom).

Fruth, Angelika (Robert Koch Institute, Enteropathogenic Bacteria and Legionella, Wernigerode, Germany).

La Ragione, Roberto M. (University of Surrey, School of Veterinary Medicine, Guildford, Surrey, United Kingdom).

Leng, Joy (University of Surrey, School of Veterinary Medicine, Guildford, Surrey, United Kingdom).

Matamoros, Sebastien (Amsterdam UMC, Department of Medical Microbiology and Infection Control, Amsterdam, the Netherlands)

Ngo, Hoa T. (Oxford University Clinical Research Unit, Vietnam; Nuffield Department of Medicine, University of Oxford, Oxford OX3 7BN, United Kingdom).

Oldenkamp, Rik (Amsterdam UMC, Department of Global Health, Amsterdam, the Netherlands).

Ritchie, Jennifer M. (University of Surrey, School of Biosciences and Medicine, Guildford, Surrey, United Kingdom).

Schultsz, Constance (Amsterdam UMC, Department of Global Health and Department of Medical Microbiology, Amsterdam, the Netherlands).

Schwarz, Stefan (Institute for Microbiology and Epizootics, Freie Universität Berlin, Berlin, Germany).

Trung, Nguyen V. (Oxford University Clinical Research Unit, Vietnam).

van der Putten, Boas (Amsterdam UMC, Department of Global Health and Department of Medical Microbiology, Amsterdam, the Netherlands).

Ugarte-Ruiz, Maria (VISAVET, Health Surveillance Center and Animal Health Department, Veterinary Faculty, Complutense University of Madrid, Spain).

\section{References}

1. Witkowska E, Korsak D, Kowalska A, Janeczek A, Kamińska A. Strain-level typing and identification of bacteria - a novel approach for SERS active plasmonic nanostructures. Anal Bioanal Chem 2018, 410:5019-5031.

2. Li W, Raoult D, Fournier P-E. Bacterial strain typing in the genomic era. FEMS Microbiol Rev 2009, 33:892-916.

3. Janda JM, Abbott SL. Bacterial identification for publication: When is enough enough? J Clin Microbio/ 2002, 40:18871891.

4. Schork NJ, Fallin D, Lanchbury JS. Single nucleotide polymorphisms and the future of genetic epidemiology. Clin Genet 2000, 58:250-264.

5. Lupski JR, Weinstock GM. Short, interspersed repetitive DNA sequences in prokaryotic genomes. J Bacterio/ 1992, $174: 4525-4529$.

6. van Belkum A, Scherer S, van Alphen L, Verbrugh H. Short-sequence DNA repeats in prokaryotic genomes. Microbiol Mol Biol Rev 1998, 62:275-293.

7. Denamur E, Clermont O, Bonacorsi S, Gordon D. The population genetics of pathogenic Escherichia coli. Nat Rev Microbiol 2021, 19:37-54. 
8. Touchon M, Hoede C, Tenaillon O, Barbe V, Baeriswyl S, Bidet P, et al. Organised genome dynamics in the Escherichia coli species results in highly diverse adaptive paths. PLOS Genet 2009, 5:e1000344.

9. Land M, Hauser L, Jun S, Nookaew I, Leuze MR, Ahn T, et al. Insights from $\mathbf{2 0}$ years of bacterial genome sequencing. Funct Integr Genomics 2015, 15:141-161.

10. Gordienko EN, Kazanov MD, Gelfand MS. Evolution of pan-genomes of Escherichia coli, Shigella spp., and Salmonella enterica. J Bacteriol 2013, 195:2786-2792.

11. Wilson GA, Bertrand N, Patel Y, Hughes JB, Feil EJ, Field D. Orphans as taxonomically restricted and ecologically important genes. Microbiology 2005, 151:2499-2501.

12. Yu G, Stoltzfus A. Population diversity of ORFan genes in Escherichia coli. Genome Biol Evol 2012, 4:1176-1187.

13. Daubin V, Ochman H. Bacterial genomes as new gene homes: The genealogy of ORFans in E. coli. Genome Res 2004, 14:1036-1042.

14. Hahn Y, Lee B. Identification of nine human-specific frameshift mutations by comparative analysis of the human and the chimpanzee genome sequences. Bioinformatics 2005, 21(Suppl 1):i186-i194.

15. Ochman H, Lawrence JG, Groisman EA. Lateral gene transfer and the nature of bacterial innovation. Nature 2000, 405:299304.

16. Toll-Riera M, Bosch N, Bellora N, Castelo R, Armengol L, Estivill X, et al. Origin of primate orphan genes: A comparative genomics approach. Mol Biol Evol 2008, 26:603-612.

17. García-Aljaro C, Ballesté E, Muniesa M. Beyond the canonical strategies of horizontal gene transfer in prokaryotes. Curr Opin Microbiol 2017, 38:95-105.

18. Medini D, Donati C, Tettelin H, Masignani V, Rappuoli R. The microbial pan-genome. Curr Opin Genet Devel 2005, 15:589594.

19. Medini D, Serruto D, Parkhill J, Relman DA, Donati C, Moxon R, et al. Microbiology in the post-genomic era. Nat Rev Microbiol 2008, 6:419-430.

20. Lukjancenko O, Wassenaar TM, Ussery DW. Comparison of 61 sequenced Escherichia coli genomes. Microb Ecol 2010, 60:708-720.

21. Pallen MJ, Wren BW. Bacterial pathogenomics. Nature 2007, 449:835-842.

22. Raskin DM, Seshadri R, Pukatzki SU, Mekalanos JJ. Bacterial genomics and pathogen evolution. Cell 2006, 124:703-714.

23. Seemann T. Prokka: rapid prokaryotic genome annotation. Bioinformatics 2014, 30:2068-2069.

24. Page AJ, Cummins CA, Hunt M, Wong VK, Reuter S, Holden MTG, et al. Roary: Rapid large-scale prokaryote pan genome analysis. Bioinformatics 2015, 31:3691-3693.

25. Altschul SF, Gish WM, W., Myers EW, Lipman DJ. Basic local alignment search tool. J Mol Bio/ 1990, 215:403-410.

26. Ye J, Coulouris G, Zaretskaya I, Cutcutache I, Rozen S, Madden TL. Primer-BLAST: A tool to design target-specific primers for polymerase chain reaction. BMC Bioinformatics 2012, 13:1-11.

27. Zangenberg G, Saiki RK, Reynolds R. Multiplex PCR: Optimization guidelines. In PCR Applications. 1st edition. Edited by Innis M, Gelfand D, Sninsky J. San Diego: Academic Press; 1999: 73-94. 
28. Svec D, Tichopad A, Novosadova V, Pfaffl MW, Kubista M. How good is a PCR efficiency estimate: Recommendations for precise and robust qPCR efficiency assessments. Biomol Detect Quantif 2015, 3:9-16.

29. Touchon M, Perrin A, Moura de Sousa JA, Vangchhia B, Burn S, O’Brien CL, et al. Phylogenetic background and habitat drive the genetic diversification of Escherichia coli. PLOS Genet 2020, 16:e1008866.

30. Vohra P, Bugarel M, Turner F, Loneragan GH, Hope JC, Hopkins J, et al. Survival of multiple Salmonella enterica serovars In Vivo via massively parallel whole-genome sequencing to predict zoonotic risk. App/ Environ Microbio/ 2018, 84:e02262-17.

31. Hamm K, Barth SA, Stalb S, Geue L, Liebler-Tenorio E, Teifke JP, et al. Experimental Infection of Calves with Escherichia coli 0104:H4 outbreak strain. Sci Rep 2016, 6:32812.

32. Nguyen JT, C. Bacterial species singled out from a diverse crowd. Nature 2020, 588:591-592.

33. Farnleitner AH, Kreuzinger N, Kavka GG, Grillenberger S, Rath J, Mach RL. Simultaneous detection and differentiation of Escherichia coli populations from environmental freshwaters by means of sequence variations in a fragment of the $\beta-d-$ glucuronidase gene. Appl Environ Microbiol 2000, 66:1340-1346.

34. Bielaszewska M, Mellmann A, Zhang W, Köck R, Fruth A, Bauwens A, Peters G, Karch H. Characterisation of the Escherichia coli strain associated with an outbreak of haemolytic uraemic syndrome in Germany, 2011: A microbiological study. Lancet Infect Dis 2011, 11:671-676.

35. Kiel M, Sagory-Zalkind P, Miganeh C, Stork C, Leimbach A, Sekse C, Mellmann A, Rechenmann F, Dobrindt U. Identification of novel biomarkers for priority serotypes of Shiga toxin-producing Escherichia coli and the development of multiplex PCR for their detection. Front Microbiol 2018, 9:1321.

\section{Tables}

Table 1. E. coli strains used in this study $(n=17)$. 


\begin{tabular}{llll} 
Strain name & Strain ID & Host & Country of origin \\
\hline ChH1 & 21225_2\#112 & Chicken & Vietnam \\
\hline ChH2 & SAP1847 & Human & UK \\
\hline ChH3 & SAP1710 & Human & UK \\
\hline C1 & IMT38565 & Cattle & Germany \\
\hline C2 & R45 & Cattle & Germany \\
\hline C3 & IMT13936 & Cattle & Germany \\
\hline C4 & IMT34414 & Cattle & Germany \\
\hline C5 & IMT10909 & Cattle & Germany \\
\hline C6 & $9475 \_4 \# 43$ & Cattle & Germany \\
\hline P1 & IMT39234 & Pig & Germany \\
\hline P2 & IMT28138 & Pig & Germany \\
\hline P3 & 39533 & Pig & Germany \\
\hline P4 & IMT38723 & Pig & Germany \\
\hline P5 & IMT38701 & Pig & Germany \\
\hline M1 & $21225 \_2 \# 178$ & Chicken & Vietnam \\
\hline M2 & 09-05726 & Human & Germany \\
\hline M3 & ZTA1601993EC & Chicken & Spain \\
\hline
\end{tabular}

Table 2. List of strain-specific PCR primers 


\begin{tabular}{|c|c|c|c|c|c|}
\hline \multirow[t]{2}{*}{ Strain ${ }^{1}$} & \multirow[t]{2}{*}{ Gene locus tag } & \multicolumn{2}{|l|}{ Primers } & \multirow{2}{*}{$\begin{array}{l}\text { Ann. } T^{\circ} \\
\left({ }^{\circ} \mathrm{C}\right)\end{array}$} & \multirow{2}{*}{$\begin{array}{l}\text { Produc } \\
\text { (bp) }\end{array}$} \\
\hline & & Forward $\left(5^{\prime} \rightarrow 3^{\prime}\right)$ & Reverse $\left(5^{\prime} \rightarrow 3^{\prime}\right)$ & & \\
\hline ChH1 & ECMDIDHM_04458 & GGAAACGGATTACTTCTACG & CTGATAGAGATTCAGTCCCC & 48 & 793 \\
\hline $\mathrm{ChH} 2$ & ANLLMAEJ_01457 & CCTACGCCACTAAACTACTG & CTATCATCACTGGAAATCCTG & 47 & 560 \\
\hline $\mathrm{ChH3}$ & PDAKODAI_04433 & GAATTACCGTCGTAGAGCAG & GGTATGGACTCAATGACAC & 47 & 970 \\
\hline C1 & KGJMKMMK_04916 & CTGCTGTTATTAATCGGCTTGG & СTCTCAAGCGTGCTTTCTATC & 51 & 886 \\
\hline $\mathrm{C} 2$ & KGPFHGEA_00668 & GTGGTGTAGAATTTATCGCATCC & AGCCATTCGATGAAACCAAG & 51 & 665 \\
\hline C3 & AMKFMELI_04046 & GGGTCGATAACTTAGCAAGC & CTTGTTTGCAGAATGCTGCG & 51 & 465 \\
\hline C4 & LCFLNNEA_01211 & GAATGGCAGGGCATACAAG & CAGACCTATGAACCTCTCCC & 52 & 522 \\
\hline C5 & GKDLAHGH_04328 & CAGGAATGACAAACCTCTCG & CCGAACCATCGATTTGTCTG & 51 & 1097 \\
\hline C6 & LDHELLGP_02401 & CTGCGGAAGAGTGTAAGTTC & CAGCGTCATCACTAAGCATT & 51 & 162 \\
\hline P1 & NIHDEHIB_01629 & GTTAGCGGAACTCCAGCGGA & CCTCAGGTGCTTACTACGTTC & 53 & 178 \\
\hline P2 & EKBHOPAC_04494 & CCAGATAGAGTCGTTTCTGC & GCAGCATTAACAGTAGGTCC & 50 & 434 \\
\hline P3 & DGMDDDKP_04627 & GGCGATACGATTTTAACACCA & GCAACGGTCTAACATTCGCTG & 50 & 347 \\
\hline P4 & LLPKFPJB_0002 & GATATAGCAAAAGCCGTTTCCTG & GCCTAGCAATAAATAACCGGTC & 51 & 564 \\
\hline P5 & HEMKEPCD_04830 & GCTCGACATATTCCGAACAG & CACAGTTCTGGTGCAATAGAG & 51 & 267 \\
\hline M1 & PJHDGJGH_02587 & GGCCATTGATAGCAGCATTG & CCGAATAATAACCATCGCC & 48 & 371 \\
\hline M2 & HDADFJHI_04570 & CAGTTATGCTGGGCTAATTG & TGCGTAATTTGCATGATATGG & 48 & 485 \\
\hline M3 & EBAAKEFM_00440 & CAGCAACGGATTGATACCTC & GCGAAGTTCTTCAATCTCC & 48 & 697 \\
\hline
\end{tabular}

${ }^{1}$ For specific strain information, see Table1.

Table 3. List of strain-specific qPCR primers 


\begin{tabular}{|c|c|c|c|c|c|}
\hline \multirow[t]{2}{*}{ Strain ${ }^{1}$} & \multirow[t]{2}{*}{ Gene locus tag } & \multicolumn{2}{|l|}{ Primers } & \multirow{2}{*}{$\begin{array}{l}\text { Ann. } \\
\mathrm{T}^{\circ} \\
\left({ }^{\circ} \mathrm{C}\right)\end{array}$} & \multirow{2}{*}{$\begin{array}{l}\text { Product } \\
\text { (bp) }\end{array}$} \\
\hline & & Forward $\left(5^{\prime} \rightarrow 3^{\prime}\right)$ & Reverse $\left(5^{\prime} \rightarrow 3^{\prime}\right)$ & & \\
\hline ChH1 & ECMDIDHM_04458 & CGCTACCAGGGACAGTACCT & TGATAGAGATTCAGTCCCCCCG & 60 & 132 \\
\hline $\mathrm{ChH} 2$ & ANLLMAEJ_01457 & ACGAATGTGACCGAGCAGAG & CAGCGTACACCGAGTAAAACC & 60 & 159 \\
\hline $\mathrm{ChH3}$ & PDAKODAI_04433 & CGGAATTACCGTCGTAGAGCAG & CAGCACGATCACCAGAATAGAAGTG & 60 & 115 \\
\hline C1 & KGJMKMMK_04916 & TGACAGCGAAAACCCAGCTC & CAGTTTGCCCCTGGATTTCC & 60 & 151 \\
\hline C2 & KGPFHGEA_00668 & TGCGTTTGCAATTTACGGCG & GCGGCTCTATCCTTTGAGTCG & 61 & 198 \\
\hline C3 & AMKFMELI_04046 & CAAACTCGACAAGAGCAACGC & AGAAGCAAAGAAACCGCCCC & 61 & 198 \\
\hline C4 & LCFLNNEA_01211 & GCGAATTGCCAAAGAAAGCCAG & TGCGGATATGCAGCAAATCTCC & 60 & 108 \\
\hline C5 & GKDLAHGH_04328 & GGCAGGGCCAAGCTTTAGTAC & GAGCTGCAAAACATGCCCATAC & 61 & 173 \\
\hline C6 & LDHELLGP_02401 & GGCTGCGGAAGAGTGTAAGTT & GCGGCTCATATTTTTCATCAGCGTC & 61 & 182 \\
\hline P1 & NIHDEHIB_01629 & CAACGAGTTAGCGGAACTCCA & СTTCTTGGCAATCAGCACAGC & 60 & 161 \\
\hline P2 & EKBHOPAC_04494 & GAGTGGAGCCATGACTTCTGC & CCAAACGCCTAATATTTCTGCGACA & 61 & 157 \\
\hline P3 & DGMDDDKP_04627 & CATGTCTTCTAATGGCGGTCGT & GTGCAACGGTCTAACATTCGCT & 60 & 123 \\
\hline P4 & LLPKFPJB_0002 & CGCAGACTCTATTGCGTCTGG & GGACTTGCGATGTAGAATCCAATC & 60 & 132 \\
\hline P5 & HEMKEPCD_04830 & AGCGGGCCGATGACAAATAC & TACAGGAAGCCGATAACCCCAC & 61 & 184 \\
\hline M1 & PJHDGJGH_02587 & CTTGCGATTGAATCTGGCAGTGT & CACACCAGCATCTATTAAGCCCTG & 60 & 168 \\
\hline M2 & HDADFJHI_04570 & CGACTGGCGCAATAACCAC & AGCACACCCGTCTTCATCATC & 60 & 151 \\
\hline M3 & EBAAKEFM_00440 & CGGCACAGGCGGAAAAAAC & CGTCACCTCGTCTCCAAACATAAAG & 61 & 250 \\
\hline uid $A^{2}$ & - & GCGAGGTACGGTAGGAGTTG & GAAGGGCGAACAGTTCCTGA & 60 & 101 \\
\hline
\end{tabular}

${ }^{1}$ For specific strain information, see Table1. ${ }^{2}$ Primers for the E. coli gene uidA (internal control).

Table 4. PCR Multiplex description 


\begin{tabular}{|c|c|c|c|c|}
\hline \multirow[t]{2}{*}{ Name of multiplex ${ }^{1}$} & \multirow[t]{2}{*}{ Strains detected ${ }^{2}$} & \multicolumn{3}{|l|}{ Conditions } \\
\hline & & Step & Temp $\left({ }^{\circ} \mathrm{C}\right)$ & Time (s) \\
\hline \multirow[t]{5}{*}{ Chicken-Human } & \multirow{5}{*}{$\begin{array}{l}\mathrm{ChH} 1 \\
\mathrm{ChH} 2 \\
\mathrm{ChH} 3\end{array}$} & Initial denaturation & 94 & 30 \\
\hline & & 30 cycles & 94 & 30 \\
\hline & & & 48 & 40 \\
\hline & & & 68 & 60 \\
\hline & & Final extension & 68 & 300 \\
\hline \multirow[t]{6}{*}{ Cattle } & $\mathrm{C} 1$ & Initial denaturation & 94 & 30 \\
\hline & $\mathrm{C} 2$ & 30 cycles & 94 & 30 \\
\hline & $\mathrm{C} 3$ & & 52 & 60 \\
\hline & $\mathrm{C} 4$ & & 68 & 70 \\
\hline & C5 & & & \\
\hline & C6 & Final extension & 68 & 300 \\
\hline \multirow[t]{5}{*}{ Pig } & $\mathrm{P} 1$ & Initial denaturation & 94 & 30 \\
\hline & P2 & 30 cycles & 94 & 30 \\
\hline & P3 & & 51 & 35 \\
\hline & $\mathrm{P} 4$ & & 68 & 40 \\
\hline & P5 & Final extension & 68 & 300 \\
\hline \multirow[t]{5}{*}{ Mix } & M1 & Initial denaturation & 94 & 30 \\
\hline & M2 & 30 cycles & 94 & 30 \\
\hline & M3 & & 49 & 40 \\
\hline & & & 68 & 45 \\
\hline & & Final extension & 68 & 300 \\
\hline
\end{tabular}

${ }^{1}$ From now on, referred to as Chicken-Human Multiplex, Cattle Multiplex, Pig Multiplex and Mix Multiplex in the text; ${ }^{2}$ Strain information displayed in detail in Table1.

Table 5. List of ORFan genes identified per strain 


\begin{tabular}{llll} 
Strain & $\mathbf{N}^{\circ}$ strain-specific genes & $\mathbf{N}^{\circ}$ of remaining ORFan genes & $\mathbf{N}^{\circ}$ genes classified as putative plasmid-encoded \\
\hline P3 & 158 & 85 & 71 \\
\hline ChH3 & 36 & 29 & 7 \\
\hline P2 & 14 & 13 & 0 \\
\hline ChH2 & 13 & 13 & 0 \\
\hline C3 & 13 & 13 & 0 \\
\hline M3 & 13 & 11 & 2 \\
\hline P5 & 11 & 11 & 0 \\
\hline M1 & 11 & 9 & 0 \\
\hline P4 & 10 & 8 & 0 \\
\hline C5 & 6 & 2 & 4 \\
\hline C4 & 5 & 5 & 0 \\
\hline ChH1 & 3 & 3 & 0 \\
\hline C2 & 3 & 3 & 0 \\
\hline P1 & 2 & 1 & 1 \\
\hline C1 & 1 & 0 & 1 \\
\hline C6 & 0 & 0 & 0 \\
\hline M2 & 0 & 0 & 0 \\
\hline
\end{tabular}

Table 6. List of $p$-values when comparing $\Delta$ Ct values for detection of experimental strains present in large intestinal content of inoculated pigs ${ }^{1}$.

\begin{tabular}{llllllll}
\multirow{2}{*}{ Experimental strains } & \multicolumn{7}{c}{ Experimental strains } \\
& & ChH1 & C1 & C2 & P2 & P3 & M1 \\
\cline { 2 - 7 } & ChH1 & & 0.2659 & 0.1330 & 0.9718 & $\mathbf{0 . 0 0 0 7}$ & 0.4575 \\
\hline C1 & & & 0.0004 & 0.7084 & 0.1751 & 0.0036 \\
\hline C2 & & & 0.0232 & $<0.0001$ & 0.9767 \\
\hline P2 & & & & $\mathbf{0 . 0 0 5 8}$ & 0.1245 \\
\hline P3 & & & & & $<0.0001$ \\
\hline
\end{tabular}

${ }^{1}$ The $p$-values $<0.05$ were considered significant (italics), $p$-values $<0.01$ were considered highly significant (bold).

\section{Figures}




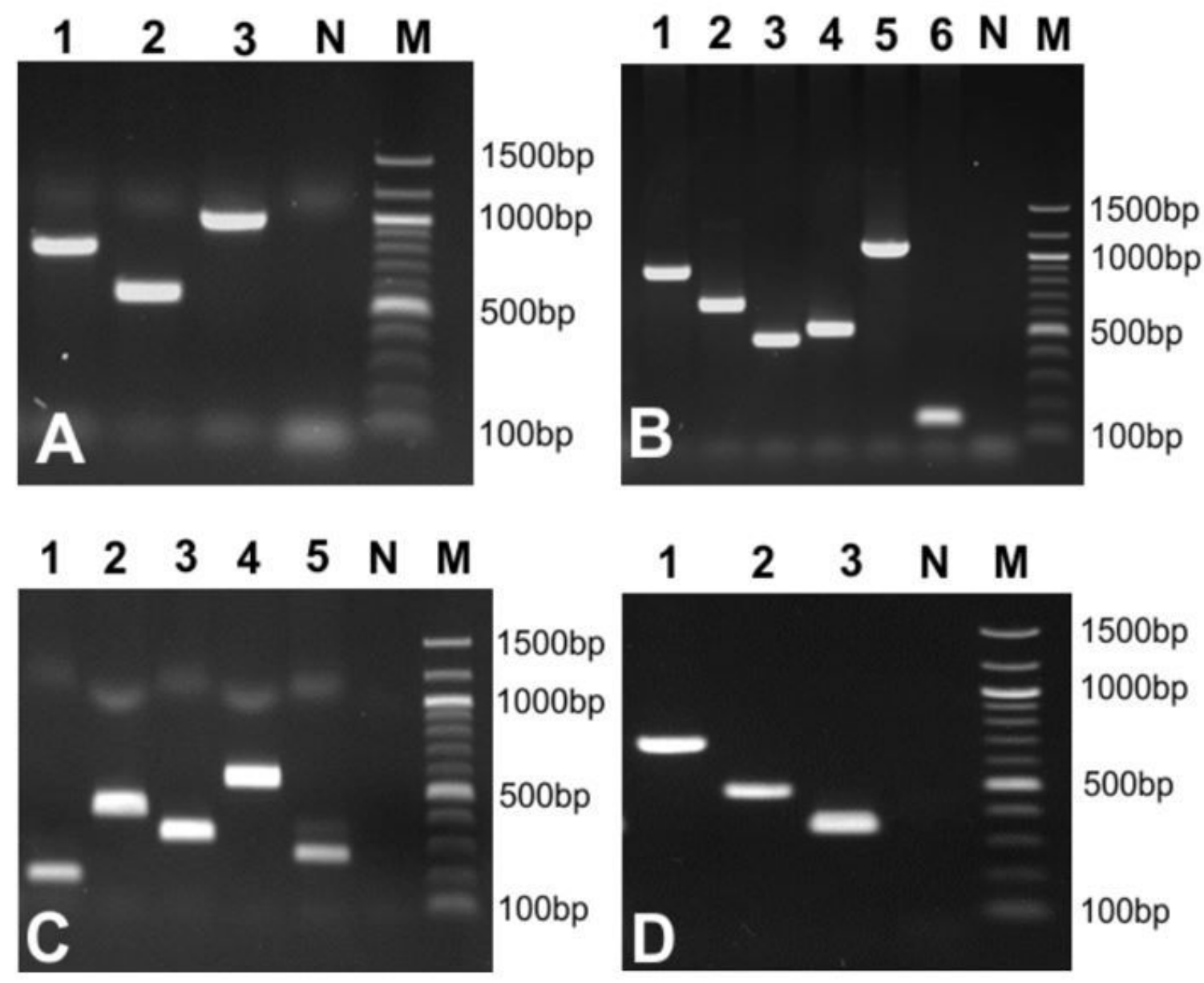

Figure 1

Results of the Multiplexes designed and their specificity for the target strains. DNA was isolated from each bacterial strain and tested with each of its corresponding multiplexes Gel A - Chicken multiplex: strains ChH1 (1), ChH2 (2), ChH3 (3); Gel B - Cattle multiplex: C1 (1), C2 (2), C3 (3), C4 (4), C5 (5), C6 (6); Gel C - Pig multiplex: strains P1 (1), P2 (2), P3 (3), P4 (4), P5 (5); Gel D Mix multiplex strains M1 (1), M2 (2), M3 (3). Lane N: Negative control; lane M: 100-bp marker (New England Biolabs Inc., Ipswich, MA, USA). 


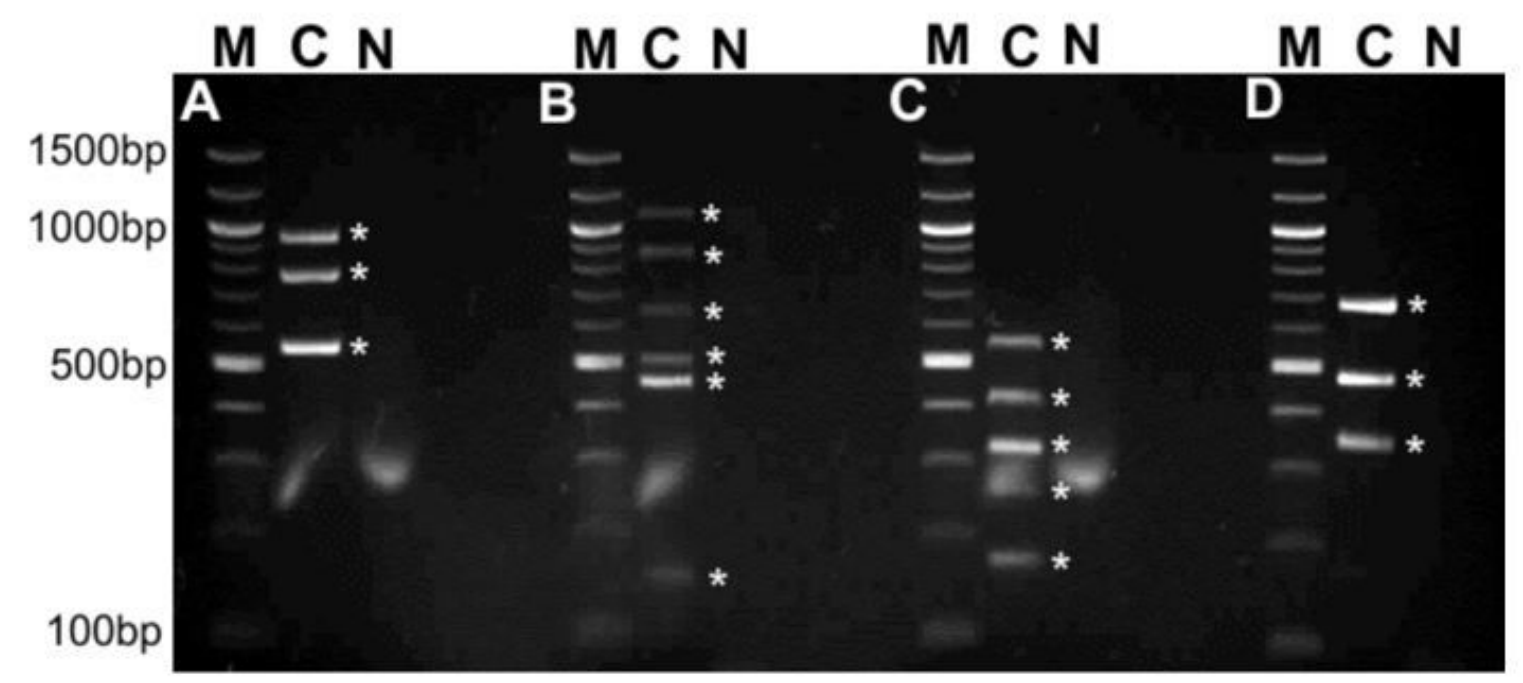

\section{Figure 2}

Results of the four multiplexes after testing DNA isolated from the inoculation cocktail. The PCR multiplexes showed that all 17 strains were present in the cocktail before animal inoculation. Lane M: 100-bp marker; lane C: DNA isolated from the experimental cocktail; lane N: negative control. Gels: A - Chicken-Human multiplex; B - Cattle multiplex; C - Pig multiplex; D - Mix multiplex. Asterisks (*) denote strain-specific PCR signals. 
Day 3 p.i.
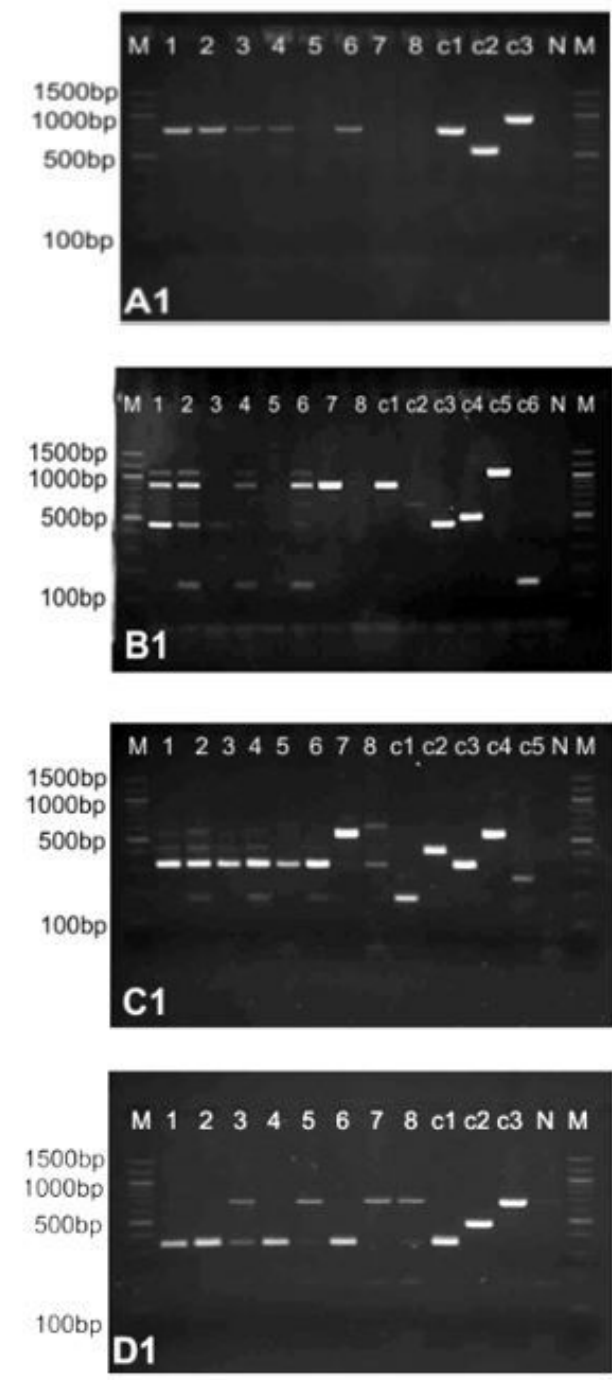

\section{Day 8 p.i.}
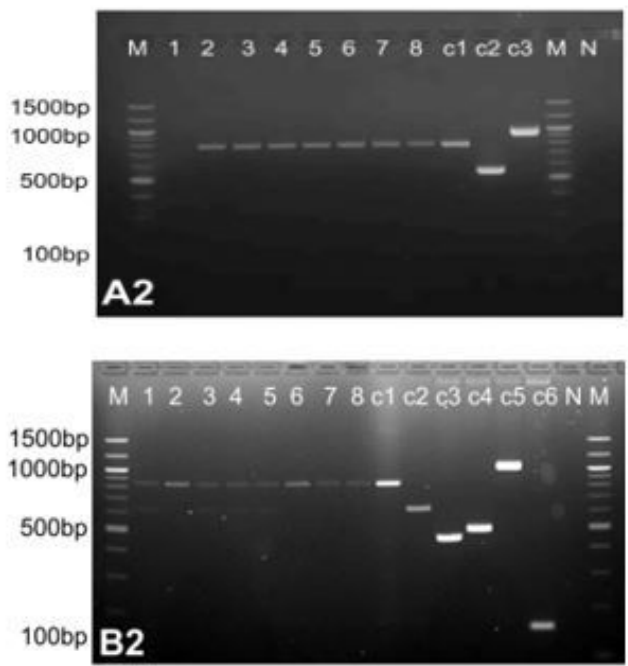

M $1223456678 \mathrm{cl}$ c2 C3C4 C5 NM

$1500 \mathrm{bp}$

$1000 \mathrm{bp}$

$500 \mathrm{bp}$

$100 \mathrm{bp}$

\section{C2}

$\begin{array}{llllllllllllllll}M & 1 & 2 & 3 & 4 & 5 & 6 & 7 & 8 & c 1 & c 2 & c 3 & \text { NM }\end{array}$

$1500 \mathrm{bp}$

$1000 \mathrm{bp}$

$500 \mathrm{bp}$

100bp

D2

\section{Figure 3}

Results of the four multiplexes after testing DNA isolated from faecal samples 3d p.i. (A1-D1) and faecal samples 8d p.i. (A2-D2). Lane M: 100-bp marker, N: Negative control, c1-c6: positive controls for each individual multiplex (controls showed in Table 1), lanes 1-8: samples isolated from each of the eight experimental pigs. Gels: A - Chicken-Human Multiplex; B - Cattle Multiplex; C

- Pig Multiplex; D - Mix Multiplex. 


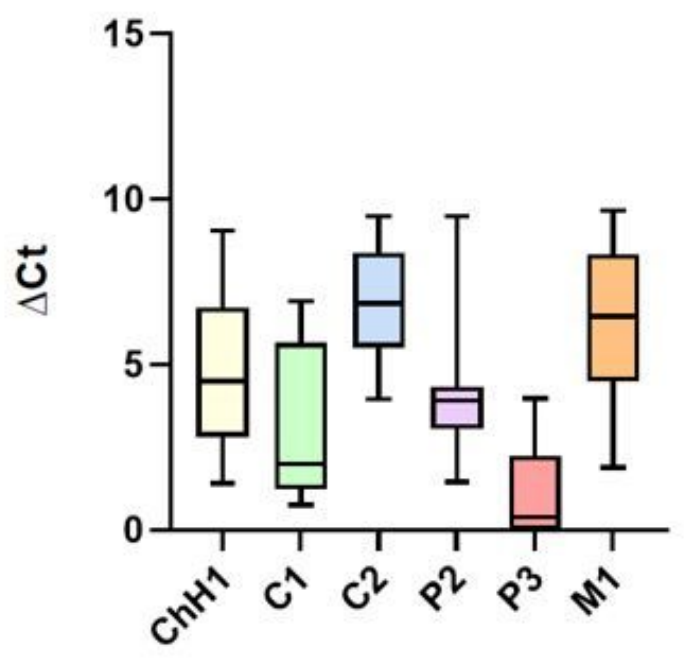

\section{Experimental strain}

\section{Figure 4}

Presence of six different experimental strains in samples taken at necropsy from the large intestine of pigs, which had been orally inoculated with a mixture of $17 \mathrm{E}$. coli strains 56 days earlier. $\Delta \mathrm{Ct}$ values obtained with samples from individual pigs are depicted on the $y$-axis (geometric mean and quartiles for all animals; $n=8$ ). Specific $p$-values for the differences in quantitative values for detecting individual strains are given in Table 6. 